\title{
Size-dependent picosecond energy relaxation in PbSe quantum dots
}

Tsuyoshi Okuno, ${ }^{\text {a) }}$ Yasuaki Masumoto, Michio Ikezawa, and Tomoyasu Ogawa

Institute of Physics, University of Tsukuba, Tsukuba, Ibaraki 305-8571, Japan

Andrey A. Lipovskii

St. Petersburg State Technical University, Polytechnicheskaja 29, St. Petersburg 195251, Russia

(Received 3 April 2000; accepted for publication 25 May 2000)

\begin{abstract}
We measured the size dependence of the energy relaxation time of PbSe quantum dots at room temperature. The radius of the dots was evaluated by small-angle x-ray scattering. We found a monotonic decrease of the relaxation time from 25 to 1 ps with a decrease of the radius $r$ from 2.9 to $1.4 \mathrm{~nm}$. The $1 / r^{3}$ dependence of the relaxation rate was indicated. (C) 2000 American Institute of Physics. [S0003-6951(00)00230-8]
\end{abstract}

Electronic and optical properties of semiconductor quantum dots have recently attracted much attention due to their interesting physical nature and potential utility for applications. ${ }^{1,2}$ Mainly researches of II-IV or III-V semiconductors have been carried out. Compared to these materials IV-VI semiconductors, such as $\mathrm{PbSe}$ or $\mathrm{PbS}$, have a larger exciton Bohr radius (46 nm for $\mathrm{PbSe}$ and $18 \mathrm{~nm}$ for $\mathrm{PbS})$. Thus, in $\mathrm{PbSe}$ or $\mathrm{PbS}$ quantum dots we can expect a large strong-confinement effect, which cannot be accessed in other materials. There are reports on the fabrication of $\mathrm{PbS}$ quantum dots with a narrow size distribution in polymers ${ }^{3}$ and silicate glasses. ${ }^{4}$ Less work has been done for PbSe. It was recently reported that $\mathrm{PbSe}$ quantum dots in phosphate glasses having various average sizes and narrow size distributions were fabricated. ${ }^{5,6}$ By using set of $\mathrm{PbS}$ and $\mathrm{PbSe}$ dots, extremely weak temperature dependence of the energy gap was studied. ${ }^{7}$

In addition to basic physical interest, $\mathrm{PbSe}$ and $\mathrm{PbS}$ dots are interesting for technology applications because their absorption peak position is tunable in the 1-3 $\mu \mathrm{m}$ range, depending on their radius. Saturable absorbers for infrared lasers by using $\mathrm{PbS}^{8,9}$ or $\mathrm{PbSe} \operatorname{dots}^{10}$ were demonstrated. Optical processing devices using possibly fast carrier lifetimes of quantum dots are other applications. Nonlinear spectroscopic studies of $\mathrm{PbS}$ dots, including decay-time measurements, were reported; ${ }^{9} 11$ however, radius dependence of the decay time has not been studied yet. There is no report on decay-time measurements or nonlinear spectroscopy of $\mathrm{PbSe}$ dots.

In this letter, we describe decay-time measurements on $\mathrm{PbSe}$ quantum dots at room temperature and report radius dependence of the decay time. In quantum-dot systems, decay time, especially at room temperature, is often determined by nonradiative carrier relaxation. The decay time is subjected to "sample quality", and it is not easy to control. However, we present here a clear dependence of the carrier lifetime against radius, that is, the smaller dots show shorter decay times.

We fabricated $\mathrm{PbSe}$ quantum dots in phosphate glasses in the manner reported in Refs. 5 and 6. Average radius is controlled by annealing temperature and duration. Figure 1

${ }^{a)}$ Electronic mail: okuno@sakura.cc.tsukuba.ac.jp shows optical absorption spectra measured at room temperature. In addition to the lowest absorption peaks (full arrows), the spectra show the second and third peaks (dashed arrows), which indicate the narrow size distribution of these samples. Depending on the annealing conditions, the lowest peaks are located between 0.84 and $1.47 \mathrm{eV}$.

In the study of the electronic-transition energy of $\mathrm{PbSe}$ quantum dots, there was a discrepancy between the calculation and the experiment. ${ }^{6}$ The calculation was performed by the envelope-function formalism using a bulk $k \cdot p$ Hamiltonian and assuming infinitely deep potential wells. ${ }^{12}$ In the experiment, the radius was obtained by transmission electron microscopy (TEM). ${ }^{6}$ The transition energy predicted by the theory was larger than that obtained from the experiment, when radius $r<3.5 \mathrm{~nm} .^{6}$ Further theoretical studies as well as experimental studies in determining radius are expected. Here, we conducted small-angle $\mathrm{x}$-ray scattering (SAXS) measurements with synchrotron radiation in order to evaluate the radius of $\mathrm{PbSe}$ dots. The x-ray scattered intensity $I(q)$ was measured as a function of the modulus of the scattering vector $q$ using a one-dimensional position sensitive detector. The vector is expressed as $q=4 \pi \sin \theta / \lambda$, where $\theta$ is half the scattering angle and $\lambda$ is the wavelength $(0.15 \mathrm{~nm})$.

Figure 2 shows Guinier plots of SAXS intensity $\{\ln [I(q)]\}$ from $\mathrm{PbSe}$ dots in phosphate glasses plotted against $q^{2}$. Energies indicated in the figure show the first

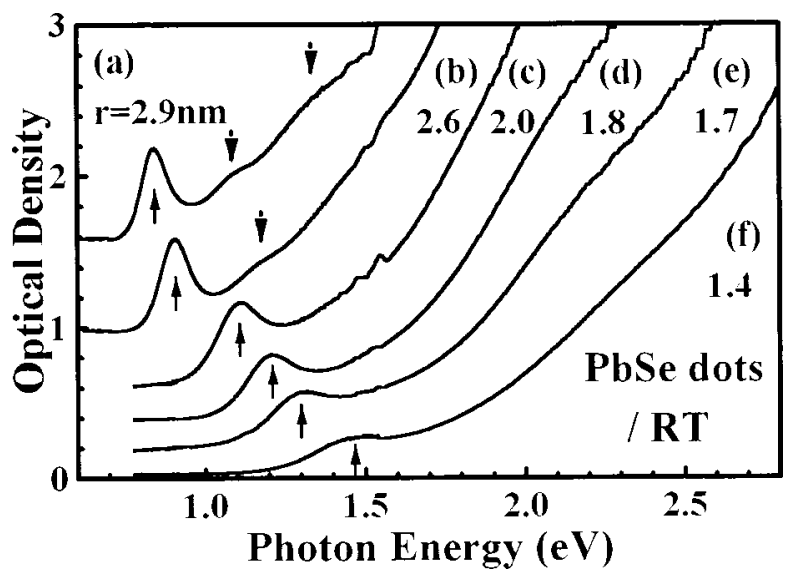

FIG. 1. Absorption spectra of PbSe quantum dots in phosphate glass at room temperature. 


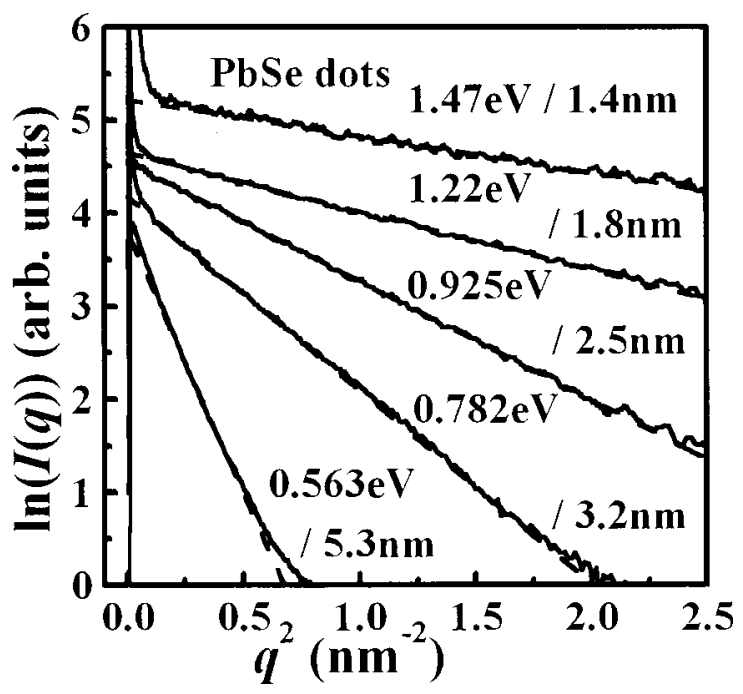

FIG. 2. Guinier plots of the small-angle x-ray scattering intensity of PbSe quantum dots in phosphate glass. The energy of the lowest absorption peak of the measured sample and the obtained radius are indicated.

transition energies determined from the lowest absorptionpeak positions of measured samples. From the Guinier plot, the average radius $r$ of $\mathrm{x}$-ray scatterers is obtained from the relation $I(q) \propto \exp \left(-q^{2} r^{2} / 5\right)$, while we assume spherical shape of the scatterers. ${ }^{13}$ We note that the Guinier plots of all the curves in Fig. 2 show straight lines, which indicate monodispersive size distribution. The radii obtained from the slopes are presented in the figure.

Squares in Fig. 3 shows the first transition energies plotted against radii obtained from SAXS. The error range of the radius was estimated from the uncertainty in the background subtraction using SAXS data of glasses not embedded with $\mathrm{PbSe}$ dots. In this figure, the results of the envelope-function calculation (dashed line) ${ }^{12}$ and the data corresponding to radii obtained from the TEM measurements (circles) ${ }^{6}$ are included. When $r>3.5 \mathrm{~nm}$, the calculation, the data using SAXS radius, and the data using TEM radius agree well. However, they begin to deviate as $r$ decreases below $3.5 \mathrm{~nm}$. Both SAXS and TEM data show slightly smaller radii than the calculation. This is supposed to be the manifestation of the finite depth of the electron and hole potential wells. At the same time, the SAXS data show larger radii than the TEM data. We do not know the reason now. The SAXS measurements reflect different electronic densities between

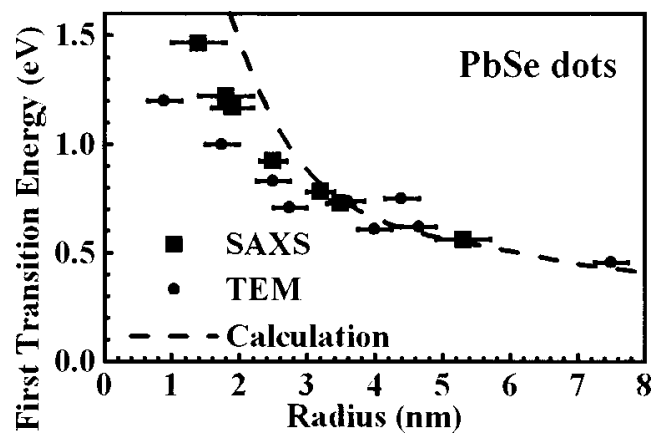

FIG. 3. The lowest transition energies for PbSe quantum dots of varying radius determined from SAXS (squares). The dashed line shows the calculated results (Ref. 12) and the circles show the data using radii determined from TEM (Ref. 6).

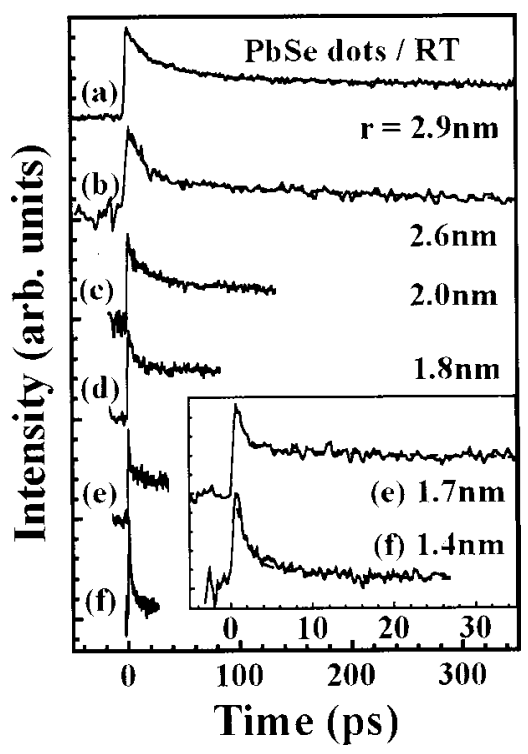

FIG. 4. Temporal changes of differential transmission in PbSe dots at room temperature. Pump photon energy was $3.1 \mathrm{eV}$ and probe photon energy was tuned to the lowest transition energy. The profiles can be fitted by twoexponential decays $\left[C_{1} \exp \left(t / \tau_{1}\right)+C_{2} \exp \left(t / \tau_{2}\right)\right]$. The obtained decay times $\tau_{1}$ and $\tau_{2}$ are (a) 25 and $1000 \mathrm{ps}$, (b) 14 and $400 \mathrm{ps}$, (c) 11 and $400 \mathrm{ps,} \mathrm{(d)}$ 5 and $1000 \mathrm{ps}$, (e) 1 and $100 \mathrm{ps}$, and (f) 1 and $30 \mathrm{ps}$. Typical errors of $\tau_{1}$ and $\tau_{2}$ were $\sim 20 \%$ and $\sim 50 \%$, respectively.

the glass matrix and the x-ray scatterers. When we assume some atoms, for example, $\mathrm{Pb}$, surrounding $\mathrm{PbSe}$ dots, ${ }^{14}$ SAXS data might show a larger radius than that of the PbSe dots. In this case, however, the agreement of the data in the region $r>3.5 \mathrm{~nm}$ seems a little difficult to understand. Even further research should be accumulated. We use SAXS radius tentatively in this letter, considering the TEM data are lacking for the largest transition energy $(>1.2 \mathrm{eV})$. In Fig. 1, the radii interpolated from the SAXS data are indicated.

We measured the energy relaxation times by using pump-and-probe experiments on $\mathrm{PbSe}$ quantum dots. A regenerative amplifier of a titanium sapphire laser was used. For the pump beam, we used the second harmonics $(3.1 \mathrm{eV})$. An optical parametric amplifier (the idler) was used to obtain the probe beam, which was resonant with the lowest transition energy (full arrows in Fig. 1). The pump beam density at the sample surface was $\sim 5 \mu \mathrm{J} / \mathrm{cm}^{2}$, and the duration was $\sim 0.2$ ps. The number of created electron-hole pairs per dot was estimated to be smaller than 1 . No noticeable change was found in relaxation profiles with different excitation densities $\left(0.5-5 \mu \mathrm{J} / \mathrm{cm}^{2}\right)$ or energy $(1.55 \mathrm{eV})$. The probe beam density was less than $1 / 10$ of the pump. The time resolution was $\sim 0.5 \mathrm{ps}$.

Figure 4 shows temporal changes of the differential transmission of $\mathrm{PbSe}$ dots at room temperature. The profiles of Figs. 4(a)-4(f) correspond to the samples shown in Figs. 1(a)-1(f), respectively. When the average radius ranges from (a) $2.9 \mathrm{~nm}$ (the largest) to (f) $1.4 \mathrm{~nm}$ (the smallest), smaller dots are found to show faster decays. The profiles can be well fitted by two-exponential decays (see the figure and the caption, and stretched exponential decays fit the data poorly). The shorter lifetime $\tau_{1}$ decreases monotonically from (a) 25 ps to (f) 1 ps. The longer lifetime $\tau_{2}$ is in the range of 30-1000 ps, which do not show clear correlation with the dot radius.

to AIP license or copyright, see http://apl.aip.org/apl/copyright.jsp 


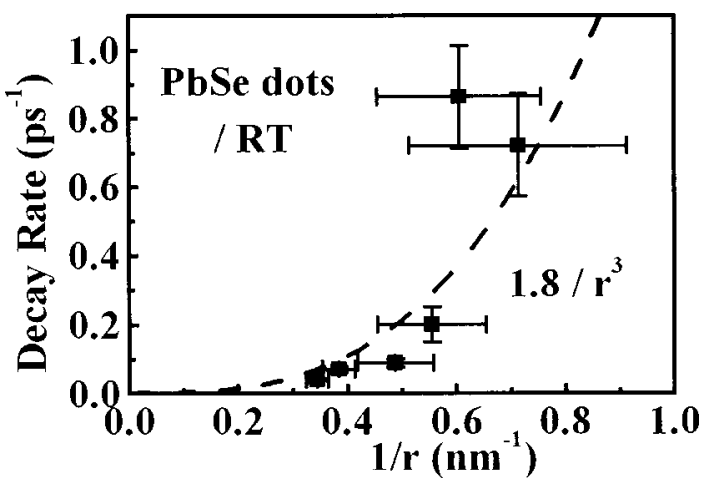

FIG. 5. Decay rate $1 / \tau_{1}$ of PbSe dots plotted against $1 / r$. The dashed line shows the $1 / r^{3}$ dependence.

When we excited these samples by an argon ion laser $(2.4 \mathrm{eV})$, exciton-related luminescence was observed between $12 \mathrm{~K}$ and room temperature. This fact indicates the high quality of these samples. The Stokes shift ranges from $\sim 100$ to $\sim 300 \mathrm{meV}$. The luminescence is thought to originate from the exciton localization at the surface or trap states. ${ }^{15}$ The luminescence intensity at room temperature was decreased to $\sim 1 / 10$ of that at $12 \mathrm{~K}$. This means the presence of the nonradiative process at room temperature.

There are several reports on carrier dynamics having two-decay components in quantum dots systems. ${ }^{15-17}$ Fast decays are often attributed to the relaxation processes of free carriers and slow decays to that of trapped carriers. Considering the presence of Stokes-shifted luminescence in $\mathrm{PbSe}$ dots, the shorter decay time $\tau_{1}$ can be ascribed to free-carrier relaxation determined by the nonradiative decay and their transition to the luminescing state. The longer decay time $\tau_{2}$ is assumed to be due to the relaxation of the localized or luminescing states, which is not discussed here because of the poorer reliability of the values.

In Fig. 5, the decay rate $1 / \tau_{1}$ is plotted against $1 / r$. If the decay rate is determined by the surface, $1 / r$ dependence (surface-to-volume ratio) is expected. ${ }^{18}$ However, the obtained dependence is $\sim 1 / r^{3}$ (dashed line). We do not have a cogent explanation of this dependence yet. Even if we use the TEM radius instead of the SAXS radius, the fit gives $1 / r^{1.7}$ dependence. A stronger dependence than $1 / r$ is clearly suggested. We consider the following as one of the possible explanations for $1 / r^{3}$ dependence. We assume the region (volume $V_{S}$ ) surrounding dots. Optically excited carriers can move to this region in which luminescing states and/or nonradiative centers are located. When $V_{S}$ is somewhat larger than the dot volume $V_{D}, V_{S}$ is assumed to be independent of dot radius. The decay rate $1 / \tau_{1}$ is thought to be the transition rate of the carriers from the dot to the surrounding region and to be proportional to $V_{S} / V_{D}$. Then we may understand $1 / r^{3}$ dependence qualitatively though more rigorous treatment is wanted. It might be possible that the clear correlation between $\tau_{1}$ and the dot radius at room temperature are coming from the extremely strong confinement in $\mathrm{PbSe}$ dots as well as the high quality of the samples.

In conclusion, we performed SAXS measurements on $\mathrm{PbSe}$ quantum dots in phosphate glasses. The obtained radii ranging from 1.4 to $3.5 \mathrm{~nm}$ correspond to those between the theoretical prediction and the reported TEM values. The size dependence of the energy relaxation time of $\mathrm{PbSe}$ dots are measured by a pump-and-probe technique at room temperature. We found a monotonic decrease of the relaxation time from 25 to $1 \mathrm{ps}$, as the radius decreased from 2.9 to $1.4 \mathrm{~nm}$. The $1 / r^{3}$ dependence of the relaxation time is indicated.

The SAXS measurements were performed at the beamline 15A of the Photon Factory, KEK, Japan, under the approval of the Photon Factory Program Advisory Committee (Proposal No. 98G071). The authors express sincere thanks to Professor Y. Amemiya, Professor H. Okuda, and Dr. H. Kamikubo for their guidance with the SAXS measurements. This work was supported by "Research for the Future" program Grant No. JSPS-RFTF97P00106 from the Japan Society for the Promotion of Science (JSPS). One of the authors (A.A.L.) was indebted to the JSPS Invitation Fellowship Programs for Research in Japan.

${ }^{1}$ A. D. Yoffe, Adv. Phys. 42, 173 (1993).

${ }^{2}$ A. P. Alivisatos, J. Phys. Chem. 100, 13226 (1996).

${ }^{3}$ M. T. Nenadovic, M. I. Comor, V. Vasic, and O. I. Micic, J. Phys. Chem. 94, 6390 (1990).

${ }^{4}$ N. F. Borrelli and D. W. Smith, J. Non-Cryst. Solids 180, 25 (1994).

${ }^{5}$ A. A. Lipovskii, E. V. Kolobkova, and V. D. Petrikov, Electron. Lett. 33, 101 (1997).

${ }^{6}$ A. Lipovskii, E. Kolobkova, V. Petrikov, I. Kang, A. Olkhovets, T. Krauss, M. Thomas, J. Silcox, F. Wise, Q. Shen, and S. Kycia, Appl. Phys. Lett. 71, 3406 (1997).

${ }^{7}$ A. Olkhovets, R.-C. Hsu, A. Lipovskii, and F. W. Wise, Phys. Rev. Lett. 81, 3539 (1998)

${ }^{8}$ P. T. Guerreiro, S. Ten, N. F. Borrelli, J. Butty, G. E. Jabbour, and N. Peyghambarian, Appl. Phys. Lett. 71, 1595 (1997).

${ }^{9}$ K. Wundke, S. Potting, J. Auxier, A. Schullzgen, N. Peyghambarian, and N. F. Borrelli, Appl. Phys. Lett. 76, 10 (2000).

${ }^{10}$ A. M. Malyarevich, I. A. Denisov, N. N. Posnov, P. V. Prokoshin, K. V. Yumashev, and A. A. Lipovskii, 15th Topical Meeting "Advanced Solid State Lasers", 13-16 February 2000, Davos, Switzerland.

${ }^{11}$ J. L. Machol, F. W. Wise, R. C. Patel, and D. B. Tanner, Phys. Rev. B 48, 2819 (1993)

${ }^{12}$ I. Kang and F. W. Wise, J. Opt. Soc. Am. B 14, 1632 (1997).

${ }^{13}$ A. Guinier and G. Fournet, Small-Angle Scattering of X-rays (Wiley, New York, 1955).

${ }^{14} \mathrm{In}$ our $\mathrm{PbSe}$-dots samples, the final concentration of $\mathrm{Pb}$ is larger than that of Se because of the high volatility of Se in the fabrication procedure.

${ }^{15}$ M. G. Bawendi, W. L. Wilson, L. Rothberg, P. J. Carroll, T. M. Jedju, M. L. Steigerwald, and L. E. Brus, Phys. Rev. Lett. 65, 1623 (1990).

${ }^{16}$ M. Tomita, T. Matsumoto, and M. Matsuoka, J. Opt. Soc. Am. B 6, 165 (1989)

${ }^{17}$ T. Okuno, H. Miyajima, A. Satake, and Y. Masumoto, Phys. Rev. B 54, 16952 (1996).

${ }^{18}$ D. M. Mittleman, R. W. Schoenlein, J. J. Shiang, V. L. Colvin, A. P. Alivisatos, and C. V. Shank, Phys. Rev. B 49, 14435 (1994). 